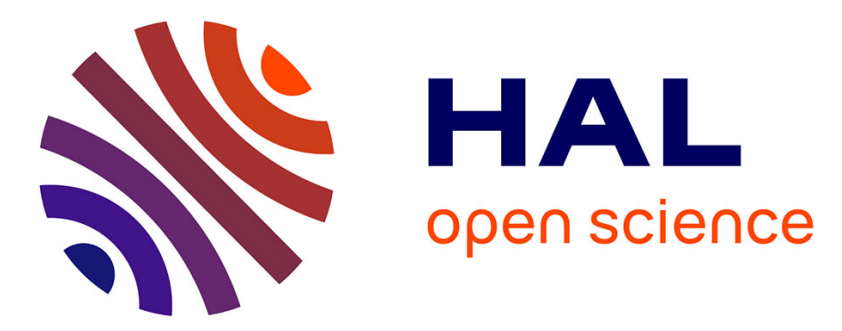

\title{
Nuclear Quantum Effects in Water Reorientation and Hydrogen-Bond Dynamics
}

David Mark Wilkins, David E. Manolopoulos, Silvio Pipolo, Damien Laage, James T. Hynes

\section{- To cite this version:}

David Mark Wilkins, David E. Manolopoulos, Silvio Pipolo, Damien Laage, James T. Hynes. Nuclear Quantum Effects in Water Reorientation and Hydrogen-Bond Dynamics. Journal of Physical Chemistry Letters, 2017, 10.1021/acs.jpclett.7b00979 . hal-01527210

\section{HAL Id: hal-01527210 https://hal.sorbonne-universite.fr/hal-01527210}

Submitted on 24 May 2017

HAL is a multi-disciplinary open access archive for the deposit and dissemination of scientific research documents, whether they are published or not. The documents may come from teaching and research institutions in France or abroad, or from public or private research centers.
L'archive ouverte pluridisciplinaire HAL, est destinée au dépôt et à la diffusion de documents scientifiques de niveau recherche, publiés ou non, émanant des établissements d'enseignement et de recherche français ou étrangers, des laboratoires publics ou privés. 
Nuclear Quantum Effects in Water Reorientation and Hydrogen-Bond Dynamics

\author{
David M. Wilkins, ${ }^{*, \dagger}$ David E. Manolopoulos, ${ }^{*, \ddagger}$ Silvio Pipolo, ${ }^{*, \boldsymbol{\top}, \S, \|}$ Damien \\ Laage, ${ }^{*}, \boldsymbol{\Phi}, \|$ and James T. Hynes ${ }^{*}, \boldsymbol{\Phi}, \perp$ \\ $\dagger$ Laboratory of Computational Science and Modeling, IMX, École Polytechnique Fédérale de \\ Lausanne, 1015 Lausanne, Switzerland \\ $\ddagger$ Physical and Theoretical Chemistry Laboratory, University of Oxford, South Parks Road, \\ Oxford OX1 3QZ, UK \\ \PASTEUR, Département de Chimie, École Normale Supérieure, UPMC Univ Paris 06, \\ CNRS, PSL Research University, 75005 Paris, France \\ $\S$ Current address: Unité de Catalyse et Chimie du Solide, Université de Lille 1, 59655 \\ Villeneuve d'Ascq, France \\ ||Sorbonne Universités, UPMC Univ Paris 06, ENS, CNRS, PASTEUR, 75005 Paris, France \\ $\perp$ Department of Chemistry and Biochemistry, University of Colorado, Boulder, CO \\ 80309-0215, USA \\ E-mail: david.wilkins@epfl.ch; david.manolopoulos@chem.ox.ac.uk; silvio.pipolo@univ-lille1.fr; \\ damien.laage@ens.fr; james.hynes@colorado.edu
}




\begin{abstract}
We combine classical and ring polymer molecular dynamics simulations with the molecular jump model to provide a molecular description of the nuclear quantum effects (NQEs) on water reorientation and hydrogen-bond dynamies in liquid $\mathrm{H}_{2} \mathrm{O}$ and $\mathrm{D}_{2} \mathrm{O}$. We show that while the net NQE is negligible in $\mathrm{D}_{2} \mathrm{O}$, it leads to a $\sim 13 \%$ acceleration in $\mathrm{H}_{2} \mathrm{O}$ dynamics compared to a classical description. Large angular jumps exchanging hydrogen-bond partners are the dominant reorientation pathway (just as in a classical description); the faster reorientation dynamics arise from the increased jump rate constant. NQEs do not change the jump amplitude distribution and no significant tunneling is found. The faster jump dynamics are quantitatively related to decreased structuring of the OO radial distribution function when NQEs are included. This is explained, via a jump model analysis, by a competition between the effects of water's librational and $\mathrm{OH}$ stretch mode zero point energies on the hydrogen-bond strength.
\end{abstract}

\title{
Graphical TOC Entry
}

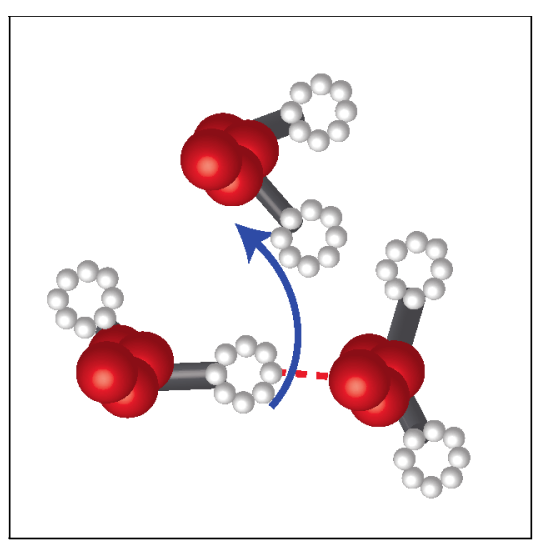

49

50

51

52

53

54

55

56

57

58

59

60 
Water reorientation and hydrogen-bond (H-bond) network rearrangements are essential for a broad range of chemical and biochemical processes in aqueous solution, including proton transfer reactions, ion transport, protein folding and ligand-biomolecule binding. ${ }^{1-4}$ Since these water motions involve displacements of hydrogens with very small mass, an explicit description of quantum mechanical zero point energy and tunneling effects may be necessary. ${ }^{5,6}$ Indeed, recent simulations have established that competing nuclear quantum effects (NQEs) on the intra- and intermolecular interactions ${ }^{7,8}$ lead to a net acceleration of water translational and rotational dynamics. ${ }^{7,9-11}$ In the case of the rotational dynamics, it has been argued ${ }^{12,13}$ on the basis of classical simulations that water reorientation proceeds via a mechanism involving sudden large angular jumps, in which H-bonding partners are exchanged during, in effect, a chemical reaction, a mechanism that is in strong contrast to the traditional Debye diffusion picture. If this jump mechanism also applies to quantum water, it could provide a detailed molecular picture of the factors responsible for the quantum rotational acceleration. But in fact, the relevance of the jump mechanism for water has been questioned, precisely because of the possible role of NQEs (see, e.g., refs 11,14).

Here we address these issues via classical molecular dynamics (MD) and - for the quantum case - thermostatted ring polymer molecular dynamics (TRPMD) ${ }^{15}$ simulations of $\mathrm{H}_{2} \mathrm{O}$ and $\mathrm{D}_{2} \mathrm{O}$ water reorientation and associated $\mathrm{H}$-bond dynamics. We show that the molecular jump mechanism ${ }^{12,13}$ remains valid when NQEs are included, and that the dynamical acceleration induced by NQEs arises from faster H-bond jump exchanges within this mechanism. Finally, we establish that the isotope and NQEs on jump dynamics can be quantitatively inferred from the changes in the oxygen-oxygen radial distribution function.

All our simulations employ the flexible q-TIP4P/F potential. ${ }^{7}$ While more sophisticated potentials are now available (e.g., MB-pol ${ }^{16}$ ), they are more expensive to evaluate; moreover since q-TIP4P/F has been shown to reproduce the experimental structural and dynamical properties of liquid water when NQEs are included, ${ }^{7}$ it is ideal for present purposes. For both $\mathrm{H}_{2} \mathrm{O}$ and $\mathrm{D}_{2} \mathrm{O}, 216$ water molecules are simulated at the experimental density ${ }^{17}$ at $298 \mathrm{~K}$ and 
propagated in 50 independent 3 ns classical NVE runs and 20 independent 500 ps quantum TRPMD $^{15}$ trajectories.

The water reorientation dynamics are probed by the orientation time-correlation functions,

$$
C_{n}(t)=\left\langle P_{n}\left[\mathbf{u}_{\mathrm{OH}}(0) \cdot \mathbf{u}_{\mathrm{OH}}(t)\right]\right\rangle
$$

where $\mathbf{u}_{\mathrm{OH}}(t)$ is the water $\mathrm{OH}$ (or OD) bond's orientation at time $t$ and $P_{n}$ is the $\mathrm{n}^{\text {th }}$ order Legendre polynomial (see SI for their calculation within the RPMD approach). These are shown for $n=1-3$ in fig 1 . Although only $C_{2}$ is experimentally accessible, ${ }^{18}$ we also consider $C_{1}$ and $C_{3}$ since different orders have been suggested ${ }^{7}$ to exhibit different NQEs. Following ref 13 , we focus on the reorientation beyond the initial sub-ps librational decay and determine the reorientation times $\tau_{n}$ by an exponential fit of $C_{n}(t)$ to $A e^{-t / \tau_{n}}$ for $4 \leq t \leq 15$ ps.
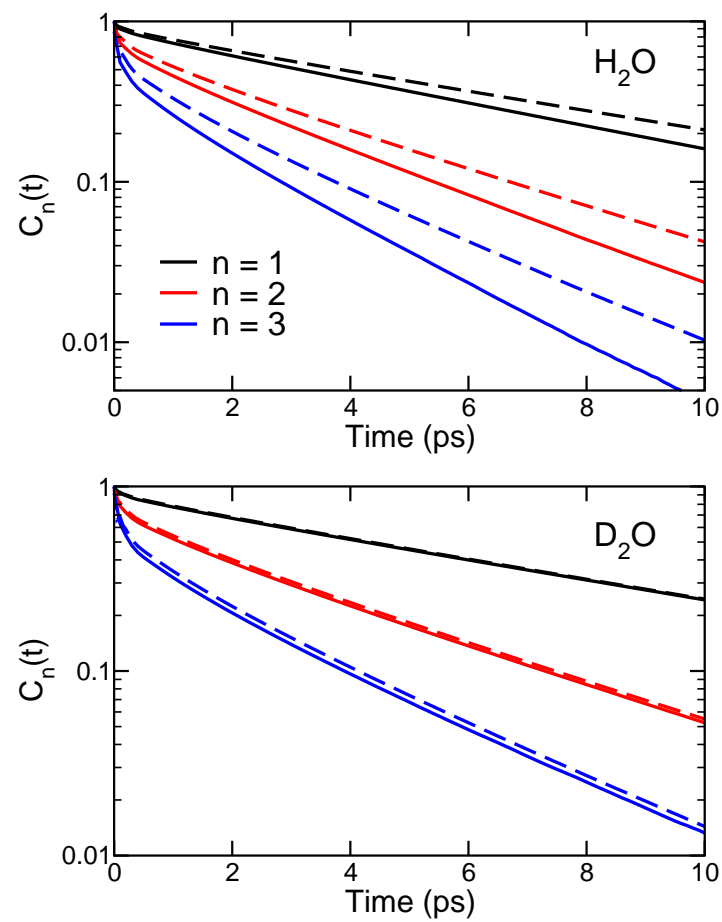

Figure 1: Orientation correlation functions $C_{n}(t)$ [eq 1] for $n=1,2,3$ for $\mathrm{H}_{2} \mathrm{O}$ (upper panel) and $\mathrm{D}_{2} \mathrm{O}$ (lower panel), from classical (dashes) and TRPMD (solid lines) simulations.

In $\mathrm{H}_{2} \mathrm{O}$, Table 1's $\tau_{n}$ times show that, in agreement with previous studies, ${ }^{7,9-11}$ NQEs 
Table 1: Reorientation times $\tau_{n}$, jump time $\tau_{0}$, inverse of frame rotational diffusion coefficient $1 / \mathrm{D}_{R}^{\text {frame }}$ and EJM reorientation times $\tau_{n}^{\mathrm{EJM}}$ (eq 2), from classical MD (cl) and TRPMD (qm) simulations, together with their ratios (all times in ps).

\begin{tabular}{c||c|c|c||c|c|c}
\hline \multicolumn{1}{c||}{} & \multicolumn{3}{c}{$\mathrm{H}_{2} \mathrm{O}$} & \multicolumn{2}{c}{$\mathrm{D}_{2} \mathrm{O}$} \\
\hline & $\mathrm{cl}$ & $\mathrm{qm}$ & $\mathrm{qm} / \mathrm{cl}$ & $\mathrm{cl}$ & $\mathrm{qm}$ & $\mathrm{qm} / \mathrm{cl}$ \\
\hline$\tau_{1}$ & $7.1(2)$ & $6.1(1)$ & $0.85(3)$ & $8.0(3)$ & $8.0(3)$ & $1.00(5)$ \\
$\tau_{2}$ & $3.7(1)$ & $3.16(7)$ & $0.86(4)$ & $4.1(1)$ & $4.12(7)$ & $1.00(4)$ \\
$\tau_{3}$ & $2.7(1)$ & $2.26(4)$ & $0.85(4)$ & $2.9(1)$ & $2.98(7)$ & $1.02(5)$ \\
\hline$\tau_{0}$ & $4.4(1)$ & $3.83(4)$ & $0.87(3)$ & $4.9(1)$ & $4.87(5)$ & $1.00(3)$ \\
$1 / D_{R}^{\text {frame }}$ & $52(1)$ & $45(1)$ & $0.86(3)$ & $56(2)$ & $59(2)$ & $1.04(5)$ \\
\hline$\tau_{1}^{\text {EIJM }}$ & $7.1(1)$ & $6.16(6)$ & $0.87(2)$ & $8.0(2)$ & $8.0(1)$ & $1.01(3)$ \\
$\tau_{2}^{\text {EJM }}$ & $3.15(7)$ & $2.73(3)$ & $0.87(2)$ & $3.50(7)$ & $3.54(5)$ & $1.01(3)$ \\
$\tau_{3}^{\text {EJM }}$ & $2.09(4)$ & $1.81(2)$ & $0.87(2)$ & $2.30(5)$ & $2.34(4)$ & $1.01(3)$ \\
\hline \hline
\end{tabular}

accelerate water reorientational dynamics. An interesting feature is that the acceleration factor $\rho_{n}=\tau_{n}^{\mathrm{qm}} / \tau_{n}^{\mathrm{cl}}$ is independent of the order $n$ of the orientational time-correlation function (tcf), and very similar to the value of 0.87 previously found ${ }^{7}$ for the NQE on the translational dynamics of the same q-TIP4P/F model, suggesting a common origin for the NQE acceleration of rotational and translational dynamics. Our results further show that the increase of $\rho_{n}$ with $n$ found in ref. 7 is actually caused by the sub-ps librational (hindered rotational) water molecular motions and not by the longer-time reorientation dynamics. As detailed in the SI, in contrast with our longer time $\tau_{n}$ values, the integrated reorientation times $\int_{0}^{\infty} C_{n}(t) \mathrm{d} t$ considered in ref. 7 include the initial librational decay. Librations make a growing contribution to the integrated times for increasing $n$; their amplitude's strong sensitivity to NQEs results in the observed increase with $n$ of those times' quantum/classical acceleration (see SI).

Turning to $\mathrm{D}_{2} \mathrm{O}$, Table 1 shows that reorientation times are not affected by NQEs. This does not imply that all $\mathrm{D}_{2} \mathrm{O}$ motions are classical, but rather that the competing NQEs on different degrees of freedom (vide infra) almost completely compensate each other; the $\mathrm{D}_{2} \mathrm{O}$ rotational dynamics can then be correctly described by classical mechanics. (In contrast with the present q-TIP4P/F potential, typical force-fields like SPC/E or TIP4P-2005 already include an effective description of NQEs for $\mathrm{H}_{2} \mathrm{O}$; thus they cannot be adapted to $\mathrm{D}_{2} \mathrm{O}$ by 
simply changing the hydrogen atoms' mass.)

Water reorientation has been argued ${ }^{12,13}$ to proceed not by the traditional Debye diffusion mechanism but rather via sudden large angular jumps when an $\mathrm{OH}$ group trades H-bond acceptors (fig 2a). As we will later pursue, these jump H-bond exchanges can be seen as a chemical reaction, breaking and making H-bonds.

Our quantum TRPMD simulations confirm that the jumps are still observed when NQEs are included (see SI), and that their mechanism is very similar to that found in classical simulations. Figure $2 \mathrm{~b}$ shows that the $\mathrm{H}_{2} \mathrm{O}$ classical and quantum-mechanical jump angle distributions are practically identical. Since the jump amplitude $\Delta \theta$ is the angle formed by the three oxygen atoms depicted in fig $2 \mathrm{a},{ }^{12,13}$ their large effective mass leads to very limited quantum fluctuations.

Within the extended jump model ${ }^{12,13}$ (EJM) description, the $\tau_{n}$ reorientation rate (inverse time) is the sum of the independent jump and frame reorientation rates. The reorientation times are thus determined by the jump time $\tau_{0}$, defined as the inverse of the jump rate constant, the jump amplitude $\Delta \theta$ and the slower H-bond complex frame reorientation time between successive H-bond jumps. The latter is close to diffusive and is approximated by $1 /\left[D_{R}^{\text {frame }} n(n+1)\right]$, where $D_{R}^{\text {frame }}$ is the frame rotational diffusion constant. When the $P(\Delta \theta)$ jump angle distribution is explicitly considered, the EJM reorientation times are ${ }^{13,19}$

$$
\begin{aligned}
\frac{1}{\tau_{n}^{\text {EJM }}=} & \frac{1}{\tau_{n}^{\text {jump }}}+\frac{1}{\tau_{n}^{\text {frame }}} \\
= & \frac{1}{\tau_{0}}\left[1-\frac{1}{2 n+1} \int_{0}^{\pi} \mathrm{d} \Delta \theta P(\Delta \theta) \frac{\sin [(2 n+1) \Delta \theta / 2]}{\sin (\Delta \theta / 2)}\right] \\
& +D_{R}^{\text {frame }} n(n+1) .
\end{aligned}
$$

We have computed the ingredients of the EJM as described in ref 13, using a Stable States approach to calculate $\tau_{0}$ and a strict geometric H-bond definition (see SI). As described in SI, very similar results are obtained with the $\mathrm{PAMM}^{20}$ probabilistic H-bond definition. In the quantum case, the ring-polymer centroids were used to calculate the jump rate constant. ${ }^{21,22}$ 
The frame rotational diffusion constant $\mathrm{D}_{R}^{\mathrm{frame}}$ was determined from the first and second order reorientation times for an intact H-bonded pair of water molecules (see SI). The resulting EJM reorientation times [eq 2] in Table 1 are seen to be in good agreement with the simulated $\tau_{n}$ values. This shows that the EJM initially suggested from an analysis of classical molecular dynamics ${ }^{12,13}$ also provides a good description of water reorientation when NQEs are included. Accordingly, we can now use the EJM to determine the origin of the isotope and nuclear quantum effects on water reorientation dynamics.

\section{a}

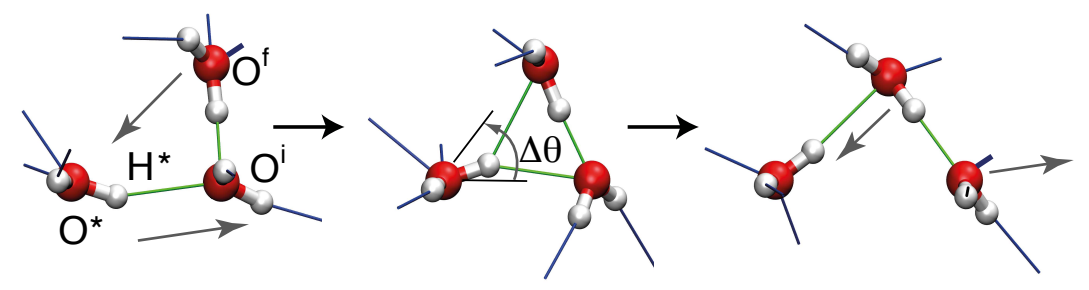

b

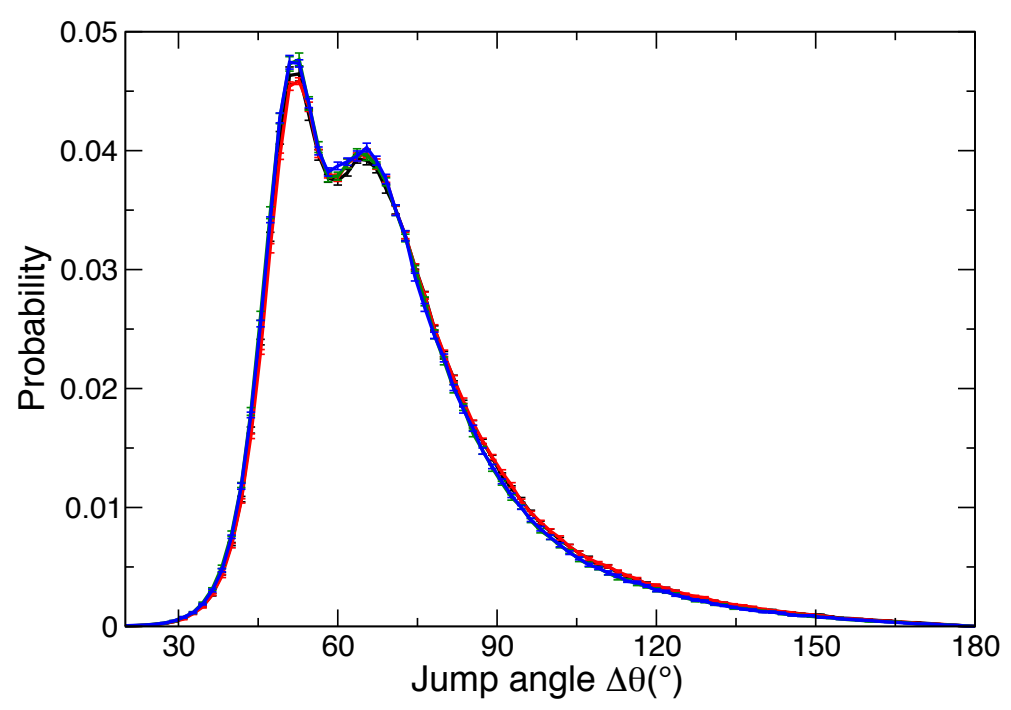

Figure 2: a) Water jump mechanism ${ }^{12,13}$ b) Distributions of jump angles $\Delta \theta$ calculated from classical $\mathrm{H}_{2} \mathrm{O}$ (black), quantum $\mathrm{H}_{2} \mathrm{O}$ (red), classical $\mathrm{D}_{2} \mathrm{O}$ (green) and quantum $\mathrm{D}_{2} \mathrm{O}$ (blue) simulations; all distributions strongly overlap.

Table 1 shows that the acceleration of $\mathrm{H}_{2} \mathrm{O}$ reorientation dynamics induced by quantum effects is essentially caused by an acceleration in the jump dynamics (the frame reorientation 
is accelerated to the same extent as the jumps, but remains much slower than the jumps in the classical and quantum descriptions). The jumps are the dominant reorientation pathway in both the classical and quantum cases, and the jump time $\tau_{0}$ exhibits exactly the same acceleration as do the $\tau_{\mathrm{n}}$ reorientation times.

We can therefore now focus on the origin of the NQE on the jump time. We immediately discard the possibility that a significant tunneling contribution could assist the water hydrogen atom's jump between the initial and final H-bond acceptors. In agreement with the conclusion of a preliminary study treating only the $\mathrm{OH}$ rotation quantum mechanically, ${ }^{13}$ our quantum simulations show that the polymer beads' distribution at the jump transition state (see SI) does not exhibit the bimodal behavior expected if tunneling were important.

In order to analyze and understand the NQE for the jump kinetics, we require a comparable jump rate formulation for both the classical and quantum situations. In the classical case, viewing, as in the previous section, the jump as a chemical reaction in which the H-bond partners of the reorienting $\mathrm{OH}$ are exchanged, leads ${ }^{13}$ to the jump rate constant expression, here written in terms of its inverse, the jump time $\tau_{0}^{\mathrm{cl}}$

$$
\tau_{0}^{\mathrm{cl}}=\frac{2 \pi}{\omega^{\mathrm{cl}}} \exp \left(\Delta G_{\mathrm{cl}}^{\ddagger} / k_{b} T\right)
$$

Here $\omega^{\mathrm{cl}}$ is the attempt frequency, i.e., the frequency of the reaction coordinate for the reactant $\left(O^{*} H^{*} \cdots O^{i}\right)$ configuration; $\Delta G_{\mathrm{cl}}^{\ddagger}$ is the activation free energy for the exchange, with the transition state (TS) defined by the $O^{*} H^{*}$ in-plane libration at the midpoint of the jump, with the two H-bonds of $H^{*}$ to $O^{i}$ and $O^{f}$ of equal length (see fig 2a). $\Delta G_{\mathrm{cl}}^{\ddagger}$ can be decomposed into contributions from different coordinates in the passage from the reactant to the TS. ${ }^{13,23}$ For the present q-TIP4P/F potential, the unstable reaction coordinate at the TS is the $O^{i} O^{*} O^{f}$ anti-symmetric stretch compressing the new $O^{*} O^{f} \mathrm{H}$-bond and expanding the old $O^{*} O^{i} \mathrm{H}$-bond. (With the SPC/E potential and other classical potentials, at the TS, the in-plane $O^{*} H^{*}$ libration has a double well potential and the reaction coordinate is this 
libration. ${ }^{13}$ ) In the reactant, the reaction coordinate is the $O^{*} O^{f}$ vibration (see SI). All other coordinates, including e.g. the $O^{*} H^{*}$ libration, the $O^{*} H^{*}$ stretch and the solvent motions are stable, transverse coordinates both in the reactant and at the TS.

Turning to the quantum description, the H-bond exchange rate constant is not conveniently couched in such explicit ingredients. However, as argued in the SI, the reaction coordinate in the reactant (the $O^{*} O^{f}$ vibration) and at the TS (the $O^{i} O^{*} O^{f}$ anti-symmetric stretch) are well approximated as classical motions. In that case, we can employ an approach analogous to that used for proton transfer reactions, ${ }^{24,25}$ and write for the quantum case

$$
\tau_{0}^{\mathrm{qu}}=\frac{2 \pi}{\omega^{\mathrm{qu}}} \exp \left(\Delta G_{\mathrm{qu}}^{\ddagger} / k_{b} T\right)
$$

where the NQEs enter in the quantum free energy barrier $\Delta G_{\mathrm{qu}}^{\ddagger}$, which includes the difference of the zero-point energies (ZPEs) of the transverse coordinates in the reactant and at the TS.

To expose the NQE's major ingredients, we will presently take the ratio of eqs 4 and 3 . But first we decompose the activation free energy in more detail. $\Delta G_{\mathrm{qu}}^{\ddagger}$ is the free energy change along the explicit H-bond-related reaction coordinate from reactant to TS, with all other, transverse, coordinates equilibrated to the reaction coordinate. (The actual dynamical path differs from this path, but this is irrelevant for the activation free energy calculation.) Since the classical and quantum mechanisms are the same, this is the free energy cost for the initial H-bond's elongation and for the final partner water molecule's approach to form the new H-bond. ${ }^{13,23}$ Finally, the SI shows that it is a good approximation to treat this barrier simply as the sum of the two independent contributions of the $O^{*} O^{i}$ and $O^{*} O^{f}$ modes,

$$
\Delta G^{\ddagger} \simeq \Delta G_{\text {elong }}^{\ddagger}+\Delta G_{\text {compr }}^{\dagger}
$$

i.e., to treat these modes as decoupled; here $\Delta G_{\text {elong,compr }}^{\ddagger}$ are respectively the free energy costs for the elongation of the initial $O^{*} O^{i}$ bond and for the compression of the $O^{*} O^{f}$ distance.

Each term in the rhs of eq 5 corresponds to the free energy cost of bringing a pair of 
water molecules from their initial, reactant state, separation to their TS separation. These contributions can thus be straightforwardly determined from the potential of mean force (pmf) along the O-O distance $W(r)$, which is related to the radial distribution function (rdf) $g(r)$ between oxygen atoms, $W(r)=-k_{B} T \ln [g(r)]$. In the reactant state configuration, $O^{i}$ lies in the first hydration shell of $O^{*}$, so that the average $O^{*}-O^{i}$ separation is the distance where the rdf exhibits its first peak $r_{\max 1}$ (fig 3). As for $O^{f}$, before the jump it lies on average in the second shell ${ }^{13}$ of $O^{*}$, and the average $O^{*}-O^{f}$ separation is therefore the radius at which the rdf exhibits its second peak, $r_{\max 2}$ (fig 3). At the jump TS, $O^{i}$ and $O^{f}$ are at the same distance from $O^{*}$, which is that of the rdf's first minimum, $r_{\min }$ (fig 3 ).

From this analysis, the free energy barrier eq 5 can thus be approximated as

$$
\Delta G^{\ddagger} \simeq\left[W\left(r_{\min }\right)-W\left(r_{\max 1}\right)\right]+\left[W\left(r_{\min }\right)-W\left(r_{\max 2}\right)\right]
$$

as illustrated in fig 3, and the classical and quantum jump times in eqs 3-4 can be estimated from the classical and quantum O-O rdfs $g^{\mathrm{cl}, \mathrm{qu}}(r)$,

$$
\tau_{0}^{\mathrm{cl}, \mathrm{qu}} \simeq \frac{2 \pi}{\omega^{\mathrm{cl}, \mathrm{qu}}} \frac{g^{\mathrm{cl}, \mathrm{qu}}\left(r_{\max 1}\right)}{g^{\mathrm{cl}, \mathrm{qu}}\left(r_{\min }\right)} \frac{g^{\mathrm{cl}, \mathrm{qu}}\left(r_{\max 2}\right)}{g^{\mathrm{cl}, \mathrm{qu}}\left(r_{\min }\right)}
$$

We have computed the $\mathrm{O}-\mathrm{O}$ rdf from both the classical and quantum simulations. As was originally found in pioneering quantum simulations of liquid water, ${ }^{5,6}$ Figure 4 shows that while NQEs do not noticeably affect the $\mathrm{D}_{2} \mathrm{O}$ rdf, they do lead to a decrease in the $\mathrm{H}_{2} \mathrm{O}$ rdf structure and thus to smaller pmf free energy barriers.

Recall from the discussion above eq 4 that the reaction coordinate comprises relative classical motions of $O^{*}$ and the oxygens of its initial and final H-bond partners; the quantum pmf therefore differs from the classical pmf because it includes ZPE contributions from all the transverse coordinates, which vary with the $\mathrm{O}-\mathrm{O}$ distance. Accordingly, we now explain the NQEs on the rdfs by considering the ZPEs of the three quantum H-bonding modes the $\mathrm{OH}$ stretch and the two librational modes - and how they change when the H-bond is 


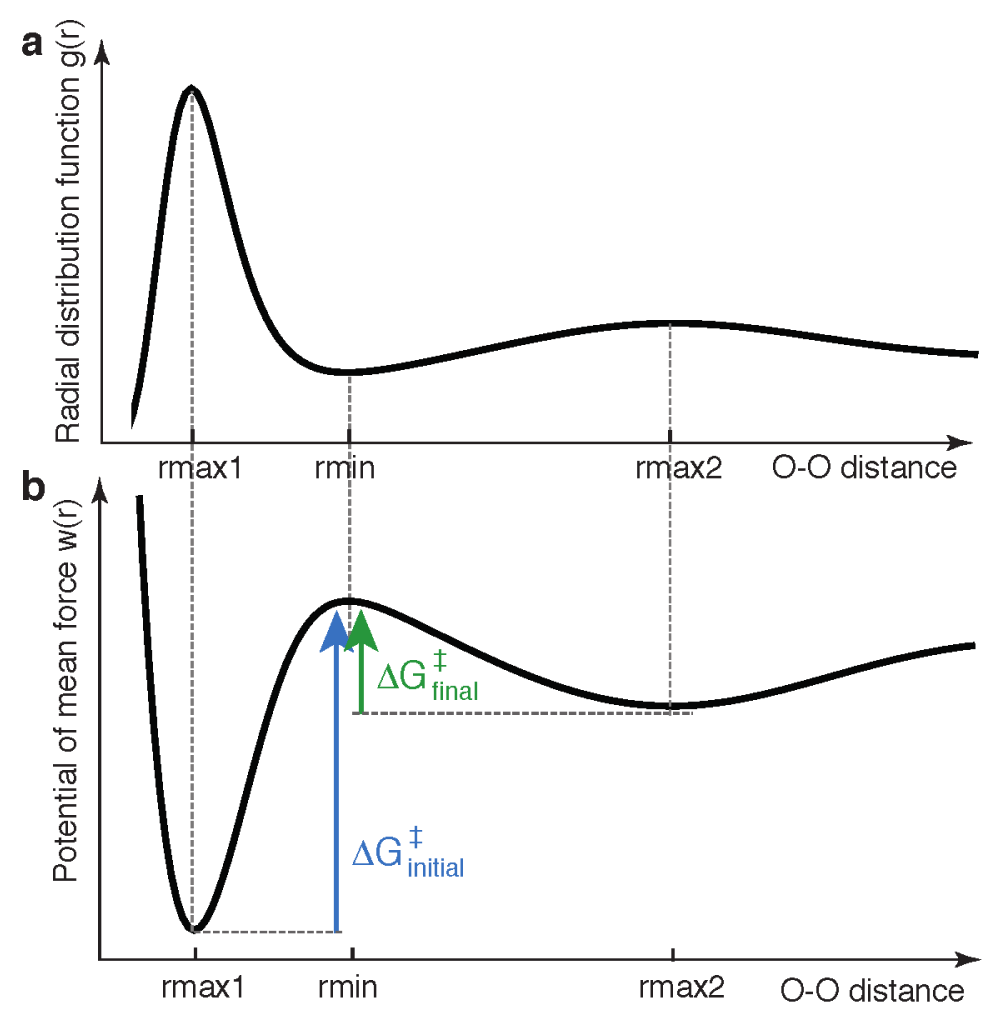

c

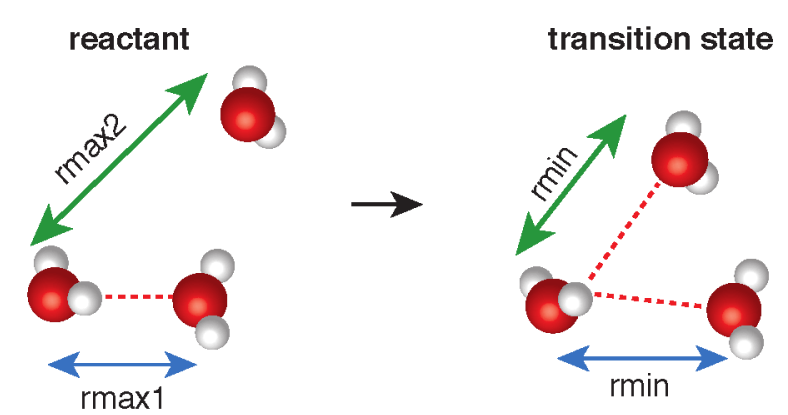

Figure 3: Schematic representation of the radial distribution function $g(r)$ (a) and potential of mean force (pmf) $W(r)$ (b) along the O-O distance between two water oxygen atoms, together with the key changes in the O-O distances during the jump (c). The $\Delta G_{\text {initial,final }}^{\ddagger}$ free energy barriers respectively associated with the elongation of the initial $O^{*} O^{i} \mathrm{H}$-bond (blue) and the compression of the $O^{*} O^{f}$ distance to the new $\mathrm{H}$-bond partner initially lying in the second hydration shell (green) are shown on the pmf. See the text.

elongated. There are competing effects here: upon H-bond elongation, the H-bond acceptor attracts the H-bond donor quantum hydrogen atom $H^{*}$ less strongly, so that with this now weaker H-bond, $H^{*}$ becomes less delocalized along the stretch mode and the $O^{*} H^{*}$ stretch ZPE increases. But this weaker H-bond situation also decreases the restoring torque on 


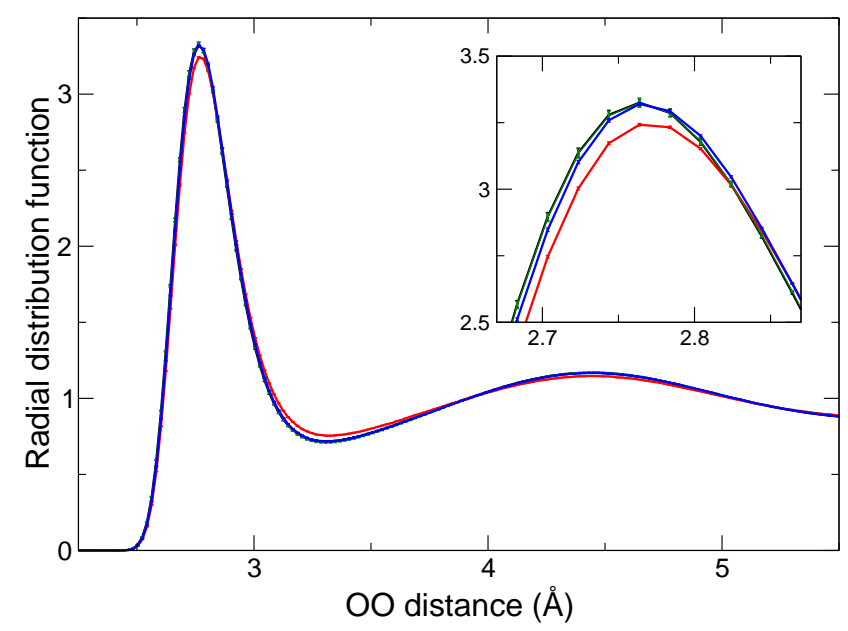

Figure 4: Radial distribution functions in classical $\mathrm{H}_{2} \mathrm{O}$ (black), quantum $\mathrm{H}_{2} \mathrm{O}$ (red), classical $\mathrm{D}_{2} \mathrm{O}$ (green) and quantum $\mathrm{D}_{2} \mathrm{O}$ (blue), where the inset focuses on the first peak region. The error bars give the Student $95 \%$ confidence interval determined from the results obtained on the set of independent trajectories. The classical $\mathrm{H}_{2} \mathrm{O}$ and $\mathrm{D}_{2} \mathrm{O}$ distributions are almost superimposed, while for the quantum distributions, there is a smaller barrier for $\mathrm{H}_{2} \mathrm{O}$ than for $\mathrm{D}_{2} \mathrm{O}$.

the donor water molecule, which becomes more delocalized along the libration coordinate, whose ZPE thus decreases. Therefore, when the O-O distance increases, the stretch ZPE increases while the librational ZPE decreases. As was originally recognized in a non-reactive context $^{7,8}$ and since been seen in several others, ${ }^{26-28}$ the NQEs on stretch and librational modes thus partly compensate each other. The overall decrease in the structure of the $\mathrm{H}_{2} \mathrm{O}$ rdf induced by NQEs (fig 4) arises from the slightly dominant effect of the librational ZPE, which decreases the free energy barriers (see SI). These competing quantum effects are thus essential to obtain a good description of the overall NQE on the dynamics. A prior study ${ }^{29}$ of quantum effects on water jump dynamics - which also indicated that the jump mechanism remained a correct water reorientation description - made two important approximations which compromise this picture of competing effects: spherical Gaussian wavepackets having the same width along the $\mathrm{OH}$ stretch and $\mathrm{OH}$ libration modes, and use of a water model with a harmonic $\mathrm{OH}$ stretch, in contrast with the present q-TIP4P/F model which accounts 
for this mode's anharmonicity.

\section{Nuclear quantum effects and isotope effects}

We now analyze the change in the jump times between two systems, conveniently labeled $a$ and $b$, which differ either by their description of nuclear dynamics - quantum vs. classical - or by their isotope $-\mathrm{H}_{2} \mathrm{O}$ vs. $\mathrm{D}_{2} \mathrm{O}$. Equations 3,4 and (in particular) 7 show that the jump time can be described as the product of three terms, arising respectively from the frequency prefactor and from the change in the free energy costs to elongate the initial bond and compress the distance to the final acceptor, so that the ratio of $a$ and $b$ jump times has a corresponding product contribution with three ingredients:

$$
\begin{aligned}
\frac{\tau_{0}^{a}}{\tau_{0}^{b}} & =\rho_{\omega} \rho_{\text {elong }} \rho_{\text {compr }} \\
\rho_{\omega} & =\omega^{\mathrm{b}} / \omega^{\mathrm{a}} \\
\rho_{\text {elong }} & =e^{\left(\Delta G_{\text {elong }}^{\ddagger a}-\Delta G_{\text {elong }}^{\ddagger b}\right) / k_{b} T}=\frac{g^{a}\left(r_{\max 1}\right)}{g^{a}\left(r_{\min }\right)} \frac{g^{b}\left(r_{\min }\right)}{g^{b}\left(r_{\max 1}\right)} \\
\rho_{\text {compr }} & =e^{\left(\Delta G_{\text {compr }}^{\ddagger a}-\Delta G_{\text {compr }}^{\ddagger b}\right) / k_{b} T}=\frac{g^{a}\left(r_{\max 2}\right)}{g^{a}\left(r_{\min }\right)} \frac{g^{b}\left(r_{\min }\right)}{g^{b}\left(r_{\max 2}\right)} .
\end{aligned}
$$

Table 2 lists the contributions of each term in eq 8 and shows that it can quantitatively predict both the isotope and nuclear quantum effects on the jump dynamics from the subtle changes in the rdf (fig 4).

We first analyze the nuclear quantum effect, i.e., the ratio between the quantum and classical jump times. The $\omega$ attempt frequency depends on the reaction coordinate reduced mass and on the pmf curvature in the reactant region. Since NQEs do not change the reduced mass and induce very small changes in the pmf curvature (fig 4 ), $\rho_{\omega} \simeq 1$. The key result shown by Table 2 is that the NQE acceleration in $\mathrm{H}_{2} \mathrm{O}$ jump dynamics is due to the remaining two factors in the product, i.e. comparable contributions from the easier elongation of the initial H-bond and from the more facile approach of the final H-bond partner. As described above, the lowering of these barriers for the $\mathrm{O}-\mathrm{O}$ motions arises from the change in the $\mathrm{OH}$ 
libration ZPE, which is partly compensated by the change in the $\mathrm{OH}$ stretch ZPE. Although the jump reaction coordinate involving the heavy $\mathrm{O}$ atoms is essentially classical, the free energy cost of its rearrangements is affected by these NQE contributions transverse to the reaction coordinate, since they change the interactions between the $\mathrm{O}$ atoms.

We now turn to the isotope effects, i.e. application of eq 8 for the ratio between the $\mathrm{H}_{2} \mathrm{O}$ and $\mathrm{D}_{2} \mathrm{O}$ jump times. The $\rho_{\omega}$ ratio is $\sqrt{18 / 20}$ since the reaction coordinate involves the motion of water molecules and not solely their hydrogen/deuterium atoms. However, Table 2 reveals that the simple picture ${ }^{30}$ assigning the isotope effect for assorted measures of water dynamics to this trivial mass effect in the present case considerably underestimates the quantum acceleration from $\mathrm{D}_{2} \mathrm{O}$ to $\mathrm{H}_{2} \mathrm{O}$. Most of this acceleration is found to arise from the change in the rdf, which leads to a decrease in the H-bond exchange free energy barriers. (Strictly speaking, one should consider the rdfs and pmfs along the distance between the water molecules' centers of mass, but as shown in the SI, these are almost indistinguishable from their analogues along the $\mathrm{O}-\mathrm{O}$ distance.) This acceleration from $\mathrm{D}_{2} \mathrm{O}$ to $\mathrm{H}_{2} \mathrm{O}$ in the NQE is due to the different reduced ZPEs of the transverse coordinates in the heavier solvent.

Table 2: Isotope and nuclear quantum effects on the H-bond jump times $\tau_{0}$ determined from our simulations and from eq 8, together with the three contributions in eq 8 (see SI).

\begin{tabular}{c||c||c|c|c|c|c}
\hline \hline & & sim & eq 8 & $\rho_{\omega}$ & $\rho_{\text {elong }}$ & $\rho_{\text {compr }}$ \\
\hline \multirow{2}{*}{$\tau_{0}^{\mathrm{q}} / \tau_{0}^{\mathrm{c}}$} & $\mathrm{H}_{2} \mathrm{O}$ & 0.87 & 0.85 & 0.99 & 0.92 & 0.93 \\
& $\mathrm{D}_{2} \mathrm{O}$ & 1.00 & 0.98 & 1.00 & 0.99 & 0.99 \\
\hline \multirow{2}{*}{$\tau_{0}^{\mathrm{H}_{2} \mathrm{O}} / \tau_{0}^{\mathrm{D}_{2} \mathrm{O}}$} & $\mathrm{qm}$ & 0.79 & 0.82 & 0.95 & 0.93 & 0.93 \\
\hline \hline
\end{tabular}

Our results thus indicate that the mass of the isotope of the reorienting group is not the only factor that determines the isotope effect on the jump time, and that the further contributions from H-bond expansion and compression in eq 8 can be important. This feature is illustrated by ultrafast spectroscopy experiments where the OD group of an HOD molecule immersed in $\mathrm{H}_{2} \mathrm{O}$ was measured to reorient faster than the $\mathrm{OH}$ group of an HOD in $\mathrm{D}_{2} \mathrm{O}\left(2.5 \pm 0.2 \mathrm{ps}\right.$ vs $\left.3.0 \pm 0.3 \mathrm{ps}^{31}\right)$. This result may seem surprising since the lighter $\mathrm{OH}$ 
group might have been expected to reorient faster than the heavier OD. Although we did not explicitly consider these isotopic mixtures here, our results show that the influence of the isotopes present in the surrounding solvent makes a very important contribution to the dynamics since they determine the free energy cost of the new partner's approach (and the frame tumbling reorientation time $\left.{ }^{12,13}\right)$.

Our study has shown that the jump picture for water reorientation applies in the nuclear quantum mechanical description as well as in the classical regime. This has allowed us to identify the molecular factors explaining the nuclear quantum and isotope effects on water H-bond and reorientation dynamics. Nuclear quantum effects lead to a moderate water dynamics acceleration, but do not affect the water reorientation mechanism, which mostly proceeds through large angular jumps, just as in the classical case. The changes in the H-bond jump dynamics are shown via a detailed jump perspective analysis to be semi-quantitatively determined by the oxygen-oxygen radial distribution function changes. Our study thus establishes a simple, robust, relationship between the liquid structure and the dynamics of H-bond jumps, which are the elementary events governing water reorientation and - since each H-bond jump induces translation of the water molecules involved - translation dynamics.

\section{Acknowledgement}

DMW thanks M. Rossi and M. Ceriotti for helpful discussions, and P. Gasparotto for advice on the PAMM algorithm. Financial support from a CNRS - Royal Society international collaborative grant (DEM, DL and JTH), the Agence Nationale de la Recherche (Grant ANR-11-BSV5-027 to DL) and NSF (Grant CHE-1112564 to JTH) is acknowledged. DL thanks for their hospitality DEM and his group at Oxford where this work was completed.

\section{Supporting Information Available}

The following files are available free of charge. Derivation of RPMD orientational time- 
correlation functions. Further discussion of the librational contribution to the integrated reorientation times, of the jump mechanism, of the PAMM H-bond analysis, of the correlated $O^{*}-O^{i}$ and $O^{*}-O^{f}$ distribution functions, and of the $\mathrm{OH}$ stretch and librational ZPEs.

\section{References}

(1) Ball, P. Water as an Active Constituent in Cell Biology. Chem Rev 2008, 108, 74-108.

(2) Laage, D.; Stirnemann, G.; Sterpone, F.; Rey, R.; Hynes, J. T. Reorientation and Allied Dynamics in Water and Aqueous Solutions. Annu Rev Phys Chem 2011, 62, 395-416.

(3) Levy, Y.; Onuchic, J. N. Water mediation in protein folding and molecular recognition. Annu Rev Biophys Biomol Struct 2006, 35, 389-415.

(4) Berkelbach, T. C.; Tuckerman, M. E. Concerted Hydrogen-Bond Dynamics in the Transport Mechanism of the Hydrated Proton: A First-Principles Molecular Dynamics Study. Phys Rev Lett 2009, 103, 238302.

(5) Kuharski, R. A.; Rossky, P. J. A quantum mechanical study of structure in liquid $\mathrm{H}_{2} \mathrm{O}$ and $\mathrm{D}_{2} \mathrm{O}$. J. Chem. Phys. 1985, 82, 5164-5177.

(6) Wallqvist, A.; Berne, B. J. Path-integral simulation of pure water. Chem. Phys. Lett. 1985, 117, 214-219.

(7) Habershon, S.; Markland, T. E.; Manolopoulos, D. E. Competing quantum effects in the dynamics of a flexible water model. J Chem Phys 2009, 131, 024501.

(8) Li, X.-Z.; Walker, B.; Michaelides, A. Quantum nature of the hydrogen bond. Proc Natl Acad Sci 2011, 108, 6369-6373.

(9) Miller, T. F.; Manolopoulos, D. E. Quantum diffusion in liquid water from ring polymer molecular dynamics. J. Chem. Phys. 2005, 123, 154504. 
(10) Paesani, F.; Iuchi, S.; Voth, G. A. Quantum effects in liquid water from an ab initio-based polarizable force field. J. Chem. Phys. 2007, 127, 074506.

(11) Paesani, F.; Yoo, S.; Bakker, H. J.; Xantheas, S. S. Nuclear Quantum Effects in the Reorientation of Water. J. Phys. Chem. Lett. 2010, 1, 2316.

(12) Laage, D.; Hynes, J. T. A Molecular Jump Mechanism of Water Reorientation. Science 2006, 311, 832-835.

(13) Laage, D.; Hynes, J. T. On the Molecular Mechanism of Water Reorientation. J. Phys. Chem. B 2008, 112, 14230-14242.

(14) Ludwig, R. The mechanism of the molecular reorientation in water. Chemphyschem 2007, 8, 44-46.

(15) Rossi, M.; Ceriotti, M.; Manolopoulos, D. E. How to remove the spurious resonances from ring polymer molecular dynamics. J. Chem. Phys. 2014, 140, 234116.

(16) Medders, G. R.; Babin, V.; Paesani, F. Development of a First-Principles Water Potential with Flexible Monomers. III. Liquid Phase Properties. J Chem Theory Comput 2014, 10, 2906-2910.

(17) Kell, G. S. Precise representation of volume properties of water at one atmosphere. $J$ Chem Eng Data 1967, 12, 66-69.

(18) Fogarty, A. C.; Duboué-Dijon, E.; Sterpone, F.; Hynes, J. T.; Laage, D. Chem. Soc. Rev. 2013, 42, 5672-5683.

(19) Boisson, J.; Stirnemann, G.; Laage, D.; Hynes, J. T. Water reorientation dynamics in the first hydration shells of $\mathrm{F}^{-}$and $\mathrm{I}^{-}$. Phys. Chem. Chem. Phys. 2011, 13, 19895-19901.

(20) Gasparotto, P.; Ceriotti, M. Recognizing molecular patterns by machine learning: An agnostic structural definition of the hydrogen bond. J. Chem. Phys. 2014, 141, 174110. 
(21) Craig, I. R.; Manolopoulos, D. E. Chemical reaction rates from ring polymer molecular dynamics. J Chem Phys 2005, 122, 084106.

(22) Craig, I. R.; Manolopoulos, D. E. A refined ring polymer molecular dynamics theory of chemical reaction rates. J Chem Phys 2005, 123, 34102.

(23) Stirnemann, G.; Laage, D. Direct Evidence of Angular Jumps During Water Reorientation Through Two-Dimensional Infrared Anisotropy. J Phys Chem Lett 2010, 1, $1511-1516$.

(24) Kiefer, P. M.; Hynes, J. T. Nonlinear Free Energy Relations for Adiabatic Proton Transfer Reactions in a Polar Environment. I. Fixed Proton DonorAcceptor Separation. J Phys Chem A 2002, 106, 1834-1849.

(25) Kiefer, P. M.; Hynes, J. T. Nonlinear Free Energy Relations for Adiabatic Proton Transfer Reactions in a Polar Environment. II. Inclusion of the Hydrogen Bond Vibration. J Phys Chem A 2002, 106, 1850-1861.

(26) Markland, T. E.; Berne, B. J. Unraveling quantum mechanical effects in water using isotopic fractionation. Proc Natl Acad Sci U S A 2012, 109, 7988-7991.

(27) Liu, J.; Andino, R. S.; Miller, C. M.; Chen, X.; Wilkins, D. M.; Ceriotti, M.; Manolopoulos, D. E. A Surface-Specific Isotope Effect in Mixtures of Light and Heavy Water. J Phys Chem C 2013, 117, 2944-2951.

(28) Wilkins, D. M.; Manolopoulos, D. E.; Dang, L. X. Nuclear quantum effects in water exchange around lithium and fluoride ions. J. Chem. Phys. 2015, 142, 064509.

(29) Ono, J.; Hyeon-Deuk, K.; Ando, K. Semiquantal molecular dynamics simulations of hydrogen-bond dynamics in liquid water using spherical gaussian wave packets. Int $J$ Quant Chem 2013, 113, 356-365. 
(30) Ceriotti, M.; Fang, W.; Kusalik, P. G.; McKenzie, R. H.; Michaelides, A.; Morales, M. A.; Markland, T. E. Nuclear Quantum Effects in Water and Aqueous Systems: Experiment, Theory, and Current Challenges. Chem Rev 2016, 116, 7529-7550.

(31) Bakker, H. J.; Rezus, Y. L. A.; Timmer, R. L. A. Molecular reorientation of liquid water studied with femtosecond midinfrared spectroscopy. J Phys Chem A 2008, 112, $11523-11534$. 